\title{
Buen planteamiento local para un problema de Cauchy asociado a una ecuación de evolución no lineal
}

\section{Luis Milla Garcia ${ }^{1}$ y Yolanda Santiago Ayald ${ }^{2}$}

Resumen: En este artículo estudiaremos el buen planteamiento local para un problema de Cauchy no lineal asociado a la ecuación diferencial KdV-Kuramoto-Sivashinsky:

$$
\left\{\begin{array}{c}
v \in C\left([0, T] ; H_{p e r}^{s}\right), s>3 / 2 \\
\partial_{t} v+\partial_{x}^{3} v+\beta\left(\partial_{x}^{4}+\partial_{x}^{2}\right) v+\left(\partial_{x} v\right)^{2}=0, \quad t>0, \beta>0 \\
v(0)=\varphi \in H_{p e r}^{s},
\end{array}\right.
$$

en los espacios infinitos dimensionales (Sobolev periódicos) $H_{p e r}^{s}$. Hacemos esto utilizando la teoría de $C_{0}$-semigrupos, principales propiedades de la transformada de Fourier en $H_{p e r}^{s}$, como las inmersiones en estos espacios y que $H_{p e r}^{s-1}$ es un álgebra de Banach, lo que nos permite justificar la presencia de la no linealidad $\left(\partial_{x} v\right)^{2}$.

Palabras claves: ecuación KdV-Kuramoto-Sivashinsky no lineal, espacios de Sobolev periódico, buen planteamiento local, teoría de Semigrupos, teoría de Fourier, Teorema del Punto fijo de Banach.

\section{Local well-posedness for a Cauchy problem associated to a non liner evolution equation}

Abstract: In this article we will study the local well-posedness for a non-linear Cauchy problem associated with the differential equation KdV- Kuramoto-Sivashinsky:

$$
\left\{\begin{array}{c}
v \in C\left([0, T] ; H_{p e r}^{s}\right), s>3 / 2 \\
\partial_{t} v+\partial_{x}^{3} v+\beta\left(\partial_{x}^{4}+\partial_{x}^{2}\right) v+\left(\partial_{x} v\right)^{2}=0, \quad t>0, \beta>0 \\
v(0)=\varphi \in H_{\text {per }}^{s},
\end{array}\right.
$$

in the infinite dimensional spaces (periodic sobolev) $H_{p e r}^{s}$. We do this using the theory of $C_{0}$-semigrupos, main properties of the Fourier transform in $H_{p e r}^{s}$, as the inmersions in these spaces and that $H_{p e r}^{s-1}$ is a Banach algebra, which allows us to justify the presence of the non-linearity $\left(\partial_{x} v\right)^{2}$.

Keywords: non linear KdV-Kuramoto-Sivashinsky equation, periodic Sobolev spaces, local well posedness, Semigroups theory, Fourier theory, Banach's fixed point theorem.

Recibido: 29/12/2020. Aceptado: 02/08/2021. Publicado online: 30/12/2021.

\footnotetext{
(CLos autores. Este artículo es publicado por la Revista PESQUIMAT de la Facultad de Ciencias Matemáticas, Universidad Nacional Mayor de San Marcos. Este es un artículo de acceso abierto, distribuido bajo los términos de la licencia Creative Commons Atribucion-No Comercia-Compartir Igual 4.0 Internacional.(http://creativecommons.org/licenses/by-nc-sa/4.0/) que permite el uso no comercial, distribución y reproducción en cualquier medio, siempre que la obra original sea debidamente citada. Para información, por favor póngase en contacto con revistapesquimat.matematica@unmsm.edu.pe.
}

${ }^{1}$ UNMSM, Facultad de Ciencias Matemáticas. e-mail: lmillag@unmsm.edu.pe

${ }^{2}$ UNMSM, Facultad de Ciencias Matemáticas, e-mail:ysantiagoa@unmsm.edu.pe 


\section{Introducción}

En el presente trabajo estudiaremos la existencia, unicidad de solución local en $H_{\text {per }}^{s}$, así como su dependencia continua respecto al dato inicial del siguiente problema

$$
\left\{\begin{array}{c}
v \in C\left([0, T] ; H_{p e r}^{s}\right), s>3 / 2 \\
\partial_{t} v+\partial_{x}^{3} v+\beta\left(\partial_{x}^{4}+\partial_{x}^{2}\right) v+\left(\partial_{x} v\right)^{2}=0, \quad t>0, \beta>0 \\
v(0)=\varphi \in H_{p e r}^{s},
\end{array}\right.
$$

donde $\beta>0$ es la constante de viscosidad, $s \in \mathbb{R}, s>3 / 2$ y $H_{\text {per }}^{s}$ es el espacio de Sobolev periódico. $\partial_{x}^{3} v$ es el término dispersivo y $\partial_{x}^{4} v$ es el término disipativo que se deducen en [9].

Este modelo describe, la propagación de ondas en medios viscosos, mas precisamente, $v$ modela la evolución de la onda deslizándose en un plano inclinado, para mayor información al respecto vea [9]. El buen planteamiento global de la parte lineal, en los espacios de Sobolev periódico $H_{\text {per }}^{s}$, para $s \in \mathbb{R}$, fue desarrollada por Yolanda en [7] y asimismo Biagoni, Bona, Iorio y Scialom en [1] desarrollaron el buen planteamiento global no lineal en $H^{s}(\mathbb{R})$, para $s \geq 1$. Tadmor en [8] obtiene resultados de buen planteamiento y estabilidad del modelo no lineal de KuramotoSivashinsky en adecuados espacios. Por tal motivo, seguimos las ideas expuestas en $[1,3,7,8]$ para estudiar el buen planteamiento local del problema de Cauchy asociado al problema no lineal (1). El presente trabajo está constituido por secciones que describiremos seguidamente:

En la sección 2 recopilamos algunas importantes desigualdades y resultados de $H_{p e r}^{s}$, teoría de puntos fijos y teoría de operadores lineales que se usarán a lo largo del trabajo. La sección 3 esta dedicada al buen planteamiento global de la parte lineal desarrollada en [7] y se consigue estimativas de regularización del semigrupo de contracción obtenido para la parte lineal. Dicha estimativa será muy útil en nuestro estudio de la parte no lineal de (1). En la sección 4 mostramos el resultado principal de nuestro trabajo, es decir, que (1) está localmente bien planteado en $H_{p e r}^{s}$, para $s>3 / 2$. Adicionalmente obtenemos más regularidad de la solución.

\section{Preliminares}

En esta sección mostramos algunos resultados que se usaron en el trabajo.

Lema 1. Supongamos que $\beta>0, \gamma>0, \beta+\gamma>1, a \geq 0, b \geq 0$ y g es una función no negativa tal que $t^{\gamma-1} g(t)$ es integrable localmente sobre $0 \leq t \leq T y$

$$
g(t) \leq a+b \int_{0}^{t}(t-\tau)^{\beta-1}(\tau)^{\gamma-1} g(\tau) d \tau
$$

en $[0, T]$. Entonces

$$
g(t) \leq a E_{\beta, \gamma}\left((b \Gamma(\beta))^{\frac{1}{\nu}} t\right)
$$

donde $\nu=\beta+\gamma-1, E_{\beta, \gamma}(s)=\sum_{m=0}^{\infty} c_{m} s^{m \nu}$ con $c_{0}=1$ y $\frac{c_{m+1}}{c_{m}}=\frac{\Gamma(m \nu+\gamma)}{\Gamma(m \nu+\gamma+\beta)}$ para $m \geq 0$.

Demostración. Ver el lema 7.1.2 en [2].

Lema 2. Sean c, $d$ constantes reales no negativos y $\tau>0$. Entonces existen $m_{\tau}>0$ y $M_{\tau}>0$ que dependen únicamente de $\tau$ satisfaciendo:

$$
m_{\tau}\left(c^{\tau}+d^{\tau}\right) \leq(c+d)^{\tau} \leq M_{\tau}\left(c^{\tau}+d^{\tau}\right) .
$$

Demostración. Citamos [3] página 205.

Teorema 1. (Teorema del Punto fijo de Banach). Sea $X=(X, d)$ un espacio métrico, donde $X \neq \varnothing$. Supongamos que $X$ es completo y sea $T: X \rightarrow X$ una contracción en $X$. Entonces $T$ tiene un único punto fijo.

Demostración. Citamos [4], página 300. 


\subsection{Espacios de Sobolev}

En esta sección introduciremos los llamados espacios de Sobolev periódico (de tipo $L^{2}$ ) y algunas de sus propiedades.

Definición 1. Sea $s \in \mathbb{R}$. El espacio de Sobolev periódico $H_{\text {per }}^{s}=H_{\text {per }}^{s}([-\pi, \pi])$ es el conjunto de todos los $f \in \mathcal{P}^{\prime}$ tal que:

$$
\|f\|_{s}^{2}=2 \pi \sum_{j \in \mathbb{Z}}\left(1+|j|^{2}\right)^{s}|\widehat{f}(j)|^{2}<\infty
$$

donde $\mathcal{P}=C_{\text {per }}^{\infty}$ es el espacio de las funciones periódicas $\varphi: \mathbb{R} \rightarrow \mathbb{C}$ las cuales son de clase $C^{\infty}$ y periódicas de periodo $2 \pi ; \mathcal{P}^{\prime}$ es el espacio de las distribuciones periódicas de periodo $2 \pi$. El conjunto $l_{s}^{2}=l_{s}^{2}(\mathbb{Z})$ es el espacio de todas las sucesiones complejas $\alpha=\left(\alpha_{j}\right)_{j \in \mathbb{Z}}$ con norma

$$
\|\alpha\|_{l_{s}^{2}}=\left[2 \pi \sum_{j \in \mathbb{Z}}\left(1+|j|^{2}\right)^{s}\left|\alpha_{j}\right|^{2}\right]^{1 / 2} .
$$

Luego $f \in H_{p e r}^{s}$ si y sólo si $(\widehat{f}(j))_{j \in \mathbb{Z}} \in l_{s}^{2}$; y por lo tanto, $\|f\|_{s}=\|\widehat{f}\|_{l_{s}^{2}}$. Como consecuencia de la identidad de Parseval, se tiene que $H_{\text {per }}^{s}$ es un espacio de Hilbert, con producto interno:

$$
\langle f \mid g\rangle_{s}=2 \pi \sum_{j \in \mathbb{Z}}\left(1+|j|^{2}\right)^{s} \widehat{f}(j) \overline{\widehat{g}(j)}
$$

En el caso $s=0, H_{p e r}^{0}$ es isomorfo isométricamente a $L_{p e r}^{2}$, el espacio de Hilbert de las funciones cuadrado integrables en el sentido de Lebesgue. En adelante $H_{p e r}^{0}$ denotará a $L_{p e r}^{2}$.

Proposición 1. Sean $s, r$ tal que $s \geq r$. Entonces $H_{\text {per }}^{s}$ está contenido continuamente en $H_{p e r}^{r}$, $\overline{H_{p e r}^{s}}\|\|_{r}=H_{p e r}^{r}$ tal que

$$
\|g\|_{r} \leq\|g\|_{s}, \forall g \in H_{p e r}^{s} .
$$

En el caso $s \geq 0, H_{\text {per }}^{s} \subset L_{\text {per }}^{2}$. Además, $\left(H_{\text {per }}^{s}\right)^{\prime}$, es isométricamente isomorfo a $H_{\text {per }}^{-s}$ para todo $s \in \mathbb{R}$. La dualidad es dada por

$$
<f, g>_{\#}=2 \pi \sum_{k \in \mathbb{Z}} \hat{f}(j) \hat{g}(j), \forall f \in H_{p e r}^{-s}, g \in H_{p e r}^{s} .
$$

Demostración. Citamos [3] página 202.

El siguiente resultado es conocido como el lema de inmersión de Sobolev. Aunque su prueba es muy sencilla, es extremadamente útil en conexión con aplicaciones de ecuaciones de evolución no lineal.

Teorema 2. Si $s>\frac{1}{2}$, entonces $H_{p e r}^{s} \hookrightarrow C_{\text {per }} y$

$$
\|f\|_{\infty} \leq\|\widehat{f}\|_{l^{1}} \leq C\|f\|_{s} \quad \forall f \in H_{p e r}^{s}
$$

Demostración. Citamos [3], página 204.

Teorema 3. Si $s>\frac{1}{2}, H_{\text {per }}^{s}$ es un álgebra de Banach. Además existe $C_{s} \geq 0$, que depende unicamente de $s$ y satisface:

$$
\|F G\|_{s} \leq C_{s}\|F\|_{s}\|G\|_{s}, \forall F, G \in H_{p e r}^{s} .
$$

Demostración. Citamos [3], página 207. 


\subsection{Familias de operadores lineales}

Daremos la noción de semigrupos de operadores lineales sobre espacios de Banach. En el presente trabajo, obtendremos un semigrupo fuertemente continuo cuyo generador infinitesimal es un operador diferencial lineal. Estos semigrupos son muy útiles al resolver ciertas ecuaciones diferenciales como el problema de Cauchy (PVI) abstracto de tipo periódico. Para más información puede consultar $[5,6]$.

Definición 2. Sea $X$ un espacio de Banach. Una familia de operadores $V(t): X \rightarrow X, s \in \mathbb{R}$ satisfaciendo

1. $V(0)=I$ donde $I$ es el operador identidad en $\mathcal{B}(X)$,

2. $V(t+r)=V(t) V(r), \forall t, r \in[0, \infty)$,

3. $\lim _{t \rightarrow 0^{+}}\|V(t) \varphi-\varphi\|=0, \forall \varphi \in X$

es llamado un semigrupo uniparamétrico fuertemente continuo en $X$ (o un $C_{0}$-semigrupo) y lo denotaremos por $\{V(t)\}_{t \geq 0}$.

Observación 1. Si $\{V(t)\}_{t \geq 0}$ es un $C_{0}$-semigrupo entonces satisface:

$$
\lim _{t \rightarrow r}\|V(t) \varphi-V(r) \varphi\|=0, \forall r \in[0, \infty), \forall \varphi \in X,
$$

esto es, la aplicación de $[0, \infty)$ a $X$ enviando $t$ a $V(t) \varphi$ es continuo.

Definición 3. Sea $\{V(t)\}_{t \geq 0}$ un $C_{0}$-semigrupo, si satisface:

$$
\|V(t)\|_{\mathcal{B}(X)} \leq 1, \forall t \in[0, \infty)
$$

entonces diremos que el semigrupo $C_{0}$ es de contracción.

\section{Parte lineal}

Examinemos la parte lineal y homogénea del problema de Cauchy KdV-K-S (1)

$$
\left\{\begin{array}{c}
v \in C\left([0, \infty) ; H_{p e r}^{s}\right) \\
\partial_{t} v+\partial_{x}^{3} v+\beta\left(\partial_{x}^{4}+\partial_{x}^{2}\right) v=0 \\
v(0)=\varphi \in H_{p e r}^{s}
\end{array}\right.
$$

donde $s>3 / 2, \beta>0$.

Asumiendo que $v$ es solución de (2). Entonces, la transformada de Fourier en la variable espacial, de la ecuación debe satisfacer

$$
\left(P_{k}\right)\left\{\begin{array}{c}
\widehat{v} \in C\left([0, \infty) ; l_{s}^{2}(\mathbb{Z})\right), \\
\partial_{t} \widehat{v}(t)=\left(i k^{3}-\beta\left(k^{4}-k^{2}\right)\right) \widehat{v}(t) \\
\widehat{v}(0)=\widehat{\varphi} \in l_{s}^{2}(\mathbb{Z})
\end{array}\right.
$$

donde $\widehat{v}(t)=(v(t, \cdot))^{\wedge}(k)$ para todo $k \in \mathbb{Z}$. Esto es una familia de ecuaciones diferenciales ordinarias: $\left(P_{k}\right)$ cuyas ecuaciones obviamente no están acopladas. Su solución es

$$
\widehat{v}(t)(k)=e^{\left(i k^{3}-\beta\left(k^{4}-k^{2}\right)\right) t} \widehat{\varphi}(k), \forall k \in \mathbb{Z}, t \in[0, \infty) .
$$

De esto se deduce que la solución, si existe, debe ser

$$
v(t)=\left(e^{\left(i(\cdot)^{3}-\beta\left((\cdot)^{4}-(\cdot)^{2}\right)\right) t} \widehat{\varphi}(\cdot)\right)^{\vee}, \forall t \in[0, \infty) .
$$


Sea

$$
Q_{\beta}(k)=i k^{3}-\beta\left(k^{4}-k^{2}\right), k \in \mathbb{Z}
$$

y definimos los operadores lineales

$$
\begin{aligned}
Q_{\beta}(D) f & =\left(-\partial_{x}^{3}-\beta\left(\partial_{x}^{4}+\partial_{x}^{2}\right)\right) f=\left(Q_{\beta}(k) \widehat{f}(\cdot)\right)^{\vee}, \\
S_{\beta}(t) f & =e^{Q_{\beta}(D) t} f=e^{\left(-\partial_{x}^{3}-\beta\left(\partial_{x}^{4}+\partial_{x}^{2}\right)\right) t} f,
\end{aligned}
$$

donde $f \in \mathcal{P}^{\prime}$. Entonces (2) puede escribirse como

$$
\left\{\begin{array}{l}
v \in C\left([0, \infty) ; H_{p e r}^{s}\right) \\
\partial_{t} v=Q_{\beta}(D) v \in H_{p e r}^{s-4} \\
v(0)=\varphi \in H_{\text {per }}^{s}
\end{array}\right.
$$

y (3) se convierte en

$$
v_{\beta}(t)=S_{\beta}(t) \varphi, t \in[0, \infty), \beta \geq 0 .
$$

Ahora estamos en condiciones de estudiar (4). Comenzamos con sus propiedades de continuidad.

Teorema 4. Sea $\beta \geq 0$ fijo. Entonces, para cualquier $s \in \mathbb{R}, t \in[0, \infty) \rightarrow S_{\beta}(t) \in \mathcal{B}\left(H_{\text {per }}^{s}\right)$ define un $C_{0}$-semigrupo de contracción en $H_{\text {per }}^{s}$. Cuando $\beta=0$, obtenemos el grupo unitario asociado a la KdV-K-S. En particular el mapeo $\varphi \in H_{\text {per }}^{s} \rightarrow S_{\beta}(t) \varphi \in H_{\text {per }}^{s}$ es continuo en el siguiente sentido. Para todo $s \in \mathbb{R}$

$$
\sup _{t \in[0, \infty)}\left\|S_{\beta}(t) \varphi_{1}-S_{\beta}(t) \varphi_{2}\right\|_{s} \leq\left\|\varphi_{1}-\varphi_{2}\right\|_{s} \text {, si } \beta>0
$$

$y$

$$
\left\|S_{0}(t) \varphi_{1}-S_{0}(t) \varphi_{2}\right\|_{s}=\left\|\varphi_{1}-\varphi_{2}\right\|_{s}, \forall t \in \mathbb{R}, \text { si } \beta=0 .
$$

Demostración. Citamos [7].

Ahora veamos la diferenciabilidad.

Teorema 5. Sea $\beta \geq 0$ fijo. Entonces

$$
\lim _{h \rightarrow 0}\left\|\frac{S_{\beta}(t+h) \varphi-S_{\beta}(t) \varphi}{h}-Q_{\beta}(D) S_{\beta}(t) \varphi\right\|_{s-4}=0
$$

converge uniformemente con respecto a $t \geq 0$.

Demostración. Citamos [7].

Finalmente obtenemos el buen planteamiento global de (4).

Corolario 1. El problema (4) esta bien planteado en $H_{\text {per }}^{s}$, para todo $r \in \mathbb{R}$. Su solución única, que depende continuamente del dato inicial, está dada por (5).

Demostración. Citamos [7].

Antes de abordar el problema no lineal estudiaremos algunas desigualdades del semigrupo $\left\{S_{\beta}(t)\right\}$.

Teorema 6. (Estimativa de regularización). Sea $\varphi \in H_{\text {per }}^{s}, s \geq 0, \lambda \geq 0, \beta>0, t>0$. Entonces existe una constante $K_{\lambda}$, dependiendo solo de $\lambda$, tal que

$$
\left\|S_{\beta}(t) \varphi\right\|_{s+\lambda} \leq K_{\lambda}\left[1+\left(\frac{1}{\beta t}\right)^{\frac{\lambda}{4}}\right]\|\varphi\|_{s},
$$

para todo $t>0$. 


\section{Demostración.}

$$
\begin{aligned}
\left\|S_{\beta}(t) \varphi\right\|_{s+\lambda}^{2} & =2 \pi \sum_{k=-\infty}^{\infty}\left(1+k^{2}\right)^{s+\lambda}\left|e^{Q_{\beta}(k) t}\right|^{2}|\widehat{\varphi}(k)|^{2} \\
& =2 \pi \sum_{k=-\infty}^{\infty}\left(1+k^{2}\right)^{s}\left(1+k^{2}\right)^{\lambda} e^{-2 \beta\left(k^{4}-k^{2}\right) t}|\widehat{\varphi}(k)|^{2} \\
& \leq\|\varphi\|_{s}^{2}\left\{\sup _{k}\left(1+k^{2}\right)^{\lambda} e^{-2 \beta t\left(k^{4}-k^{2}\right)}\right\}
\end{aligned}
$$

usando el lema $2 \operatorname{con} \tau:=\lambda>0$

$$
\begin{aligned}
& \leq C_{\lambda}\|\varphi\|_{s}^{2}\left\{\sup _{k \in \mathbb{Z}}\left(e^{-2 \beta t\left(k^{4}-k^{2}\right)}+k^{2 \lambda} e^{-2 \beta t\left(k^{4}-k^{2}\right)}\right)\right\} \\
& \left.\leq C_{\lambda}\|\varphi\|_{s}^{2}\left\{1+\sup _{k \in \mathbb{Z}} k^{2 \lambda} e^{-2 \beta t\left(k^{4}-k^{2}\right)}\right)\right\} \\
& \left.\leq C_{\lambda}\|\varphi\|_{s}^{2}\left\{1+\sup _{|k| \geq 2} k^{2 \lambda} e^{-2 \beta t\left(k^{4}-k^{2}\right)}\right)\right\} \\
& \leq C_{\lambda}\|\varphi\|_{s}^{2}\left\{1+\sup _{k \geq 2} k^{2 \lambda} e^{-\beta t k^{4}}\right\} .
\end{aligned}
$$

Por lo tanto, la desigualdad (7) se sigue de (8) y de observar que la función $g(r)=r^{2 \lambda} e^{-\beta t r^{4}}, r>0$ alcanza su valor máximo en $r=\sqrt[4]{\frac{\lambda}{2 \beta t}}$.

Observación 2. El teorema probado (6) justifica la regularidad de la solución de la parte lineal y homogénea (2) usando las normas de Sobolev. Otra forma alternativa se uso en [7].

Teorema 7. La función $t \in[\epsilon, \infty) \rightarrow S_{\beta}(t) \varphi=e^{Q_{\beta}(D)(t)} \varphi, \beta>0$, es uniformemente continua con respecto a $H_{\text {per }}^{s+\lambda}$ para todo $\lambda \geq 0$ y todo $\epsilon>0$. En efecto, existe una constante $K(\lambda, \epsilon, \beta, 4)$ tal que

$$
\left\|S_{\beta}(t) \varphi-S_{\beta}(\tau) \varphi\right\|_{s+\lambda} \leq K|t-\tau|\|\varphi\|_{s}
$$

para todo $t, \tau \geq \epsilon$. En particular, $S_{\beta}(\cdot) \varphi \in C((0, \infty) ; \mathcal{P})$.

Demostración. Sin perdida de generalidad, consideremos $t \geq \tau \geq \epsilon$. Entonces,

$$
\begin{aligned}
\left\|S_{\beta}(t) \varphi-S_{\beta}(\tau) \varphi\right\|_{s+\lambda}^{2} & =2 \pi \sum_{k=-\infty}^{\infty}\left(1+k^{2}\right)^{s+\lambda}\left|e^{Q_{\beta}(k) t}-e^{Q_{\beta}(k) \tau}\right|^{2}|\widehat{\varphi}(k)|^{2} \\
& =2 \pi \sum_{k=-\infty}^{\infty}\left(1+k^{2}\right)^{s+\lambda} e^{-2 \beta\left(k^{4}-k^{2}\right) \tau}\left|e^{Q_{\beta}(k)(t-\tau)}-1\right|^{2}|\widehat{\varphi}(k)|^{2} .
\end{aligned}
$$

El teorema del valor medio implica que

$$
\left|e^{Q_{\beta}(k)(t-\tau)}-1\right| \leq\left|Q_{\beta}(k)\right||t-\tau| \text {. }
$$


En consecuencia,

$$
\begin{aligned}
& \left\|S_{\beta}(t) \varphi-S_{\beta}(\tau) \varphi\right\|_{s+\lambda}^{2} \\
& \leq(1+\beta)^{2}|t-\tau|^{2} 2 \pi \sum_{k=-\infty}^{\infty}\left(1+k^{2}\right)^{s+\lambda} e^{-2 \beta\left(k^{4}-k^{2}\right) \tau}\left|k^{4}\right|^{2}|\widehat{\varphi}(k)|^{2} \\
& \leq(1+\beta)^{2}|t-\tau|^{2} 2 \pi \sum_{k=-\infty}^{\infty}\left(1+k^{2}\right)^{s}\left(1+k^{2}\right)^{\lambda} e^{-2 \beta\left(k^{4}-k^{2}\right) \tau}\left(1+k^{2}\right)^{4}|\widehat{\varphi}(k)|^{2} \\
& \leq(1+\beta)^{2}|t-\tau|^{2}\|\varphi\|_{s}^{2}\left\{\underset{k}{\sup }\left(1+k^{2}\right)^{\lambda+4} e^{-2 \beta\left(k^{4}-k^{2}\right) \tau}\right\}
\end{aligned}
$$

usando el lema 2

$$
\begin{aligned}
& \leq C_{\lambda}(1+\beta)^{2}|t-\tau|^{2}\|\varphi\|_{s}^{2}\left\{1+\sup _{k} k^{2(\lambda+4)} e^{-2 \beta \tau\left(k^{4}-k^{2}\right)}\right\} \\
& \leq C_{\lambda}(1+\beta)^{2}|t-\tau|^{2}\|\varphi\|_{s}^{2}\left\{1+\sup _{|k| \geq 2} k^{2(\lambda+4)} e^{-\beta \epsilon k^{4}}\right\} .
\end{aligned}
$$

Considerando la función $g(r)=r^{2 \lambda+8} e^{-\beta \epsilon r^{4}}, r>0$, que alcanza su máximo en $r=\sqrt[4]{\frac{\lambda+4}{2 \beta \epsilon}}$, obtenemos

$$
\left\|S_{\beta}(t) \varphi-S_{\beta}(\tau) \varphi\right\|_{s+\lambda} \leq K(\lambda, \epsilon, \beta, 4)|t-\tau|\|\varphi\|_{s}
$$

\section{Teoría local no lineal}

En esta sección estableceremos la existencia, unicidad y dependencia continua respecto al dato inicial de la solución en $H_{p e r}^{s}$ del problema de Cauchy abstracto no lineal.

$$
\left\{\begin{array}{c}
v \in C\left([0, T] ; H_{\text {per }}^{s}\right) \\
\partial_{t} v+\partial_{x}^{3} v+\beta\left(\partial_{x}^{4}+\partial_{x}^{2}\right) v+\left(\partial_{x} v\right)^{2}=0, \quad t>0, \beta>0 \\
v(0)=\varphi \in H_{p e r}^{s}
\end{array}\right.
$$

para $s>3 / 2$. Observe que el término no lineal tiene sentido desde que $H_{\text {per }}^{s}$ es un álgebra de Banach. Empezamos estudiando dicho término.

Proposición 2. Sea $v \in C\left([0, T] ; H_{\text {per }}^{s}\right)$. Entonces $\left(\partial_{x} v\right)^{2} \in C\left([0, T] ; H_{\text {per }}^{s-1}\right)$, para $s>\frac{3}{2}$.

\section{Demostración.}

$\left(\partial_{x} v\right)^{2}(t) \in H_{p e r}^{s-1}, s>3 / 2$ se desprende desde que $H_{p e r}^{s-1}$ es un álgebra de Banach para $s>3 / 2$. La continuidad de $\left[\partial_{x}(\cdot)\right]^{2}$ se obtiene gracias al teorema 7 . En efecto

$$
\begin{aligned}
\left\|\left(\partial_{x} v\right)^{2}(t)-\left(\partial_{x} v\right)^{2}(\tau)\right\|_{s-1} & \leq\left\|\left(\partial_{x} v\right)(t)+\left(\partial_{x} v\right)(\tau)\right\|_{s-1}\left\|\left(\partial_{x} v\right)(t)-\left(\partial_{x} v\right)(\tau)\right\|_{s-1} \\
& \leq \sup _{t, \tau \in[0, T]}\left(\|v(t)\|_{s}+\|v(\tau)\|_{s}\right)\|v(t)-v(\tau)\|_{s} \\
& \leq 2 M K \mid t-\tau\|\| \varphi \|_{s}
\end{aligned}
$$

donde $M=\max _{t \in[0, T]}\|v(t)\|_{s}$. Así hemos probado que $\left[\partial_{x} v(\cdot)\right]^{2} \in C\left([0, T] ; H_{p e r}^{s-1}\right)$. 
De la proposición 2 resulta que, si, para $s>3 / 2$ y algún $T>0, v \in C\left([0, T] ; H_{\text {per }}^{s}\right)$ es solución de 110 , entonces usando el lema de inmersión $\partial_{t} v \in C\left([0, T] ; H_{\text {per }}^{s-4}\right)$, con la derivada del tiempo dada por

$$
\lim _{h \rightarrow 0}\left\|\frac{v(t+h)-v(t)}{h}+\partial_{x}^{3} v+\beta\left(\partial_{x}^{4} v+v \partial_{x}^{2} v\right)+\left(\partial_{x} v\right)^{2}\right\|_{s-4}=0
$$

donde el limite es tomado lateral por derecha en $t=0 \mathrm{y}$ por la izquierda en $t=T$.

Para obtener una solución formal de (10), utilizamos el método de variación de parámetro. Tomando la transformada de Fourier de la ecuación diferencial parcial y la condición inicial, obtenemos el problema

$$
\left\{\begin{aligned}
\partial_{t} \widehat{v}(t, k)-\left(i k^{3}-\beta\left(k^{4}-k^{2}\right)\right) \widehat{v}(t, k)+\left[\left(\partial_{x} v\right)^{2}\right]^{\wedge}(t, k) & =0, \\
\widehat{v}(t, k) & =\widehat{\varphi}(k) .
\end{aligned}\right.
$$

Multiplicando esta ecuación diferencial ordinaria por un factor integrante adecuado, integrando sobre $[0, t]$, usando la condición inicial y tomando la transformada de Fourier inversa, obtenemos

$$
v(t)=S_{\beta}(t) \varphi-\int_{0}^{t} S_{\beta}\left(t-t^{\prime}\right)\left(\partial_{x} v\right)^{2}\left(t^{\prime}\right) d t^{\prime}
$$

donde $\left\{S_{\beta}(t)\right\}$ es el semigrupo introducido en la sección anterior. El siguiente teorema muestra que la ecuación integral (11) es equivalente al problema de Cauchy 10.

Teorema 8. Sea $v \in\left(C[0, T] ; H_{\text {per }}^{s}\right)$ solución de (10). Entonces v satisface (11). Inversamente, si $v \in\left(C[0, T] ; H_{\text {per }}^{s}\right)$ es una solución de $[11)$, entonces $v \in\left(C^{1}[0, T] ; H_{\text {per }}^{s}\right)$ y satisface 10$)$.

Demostración. Sea $v \in C\left([0, T] ; H_{p e r}^{s}\right)$.

1. Supongamos primero que $v \in C^{1}\left([0, T] ; H_{p e r}^{s-4}\right)$ y que es solución de $[10)$. Por el teorema 5 , se sigue que

$$
\begin{aligned}
\partial_{t^{\prime}}\left(S_{\beta}\left(t-t^{\prime}\right) v\left(t^{\prime}\right)\right) & =-Q_{\beta}(D) S_{\beta}\left(t-t^{\prime}\right) v\left(t^{\prime}\right)+S_{\beta}\left(t-t^{\prime}\right) \partial_{t^{\prime}} v\left(t^{\prime}\right) \\
& =S_{\beta}\left(t-t^{\prime}\right)\left[-Q_{\beta}(D) v\left(t^{\prime}\right)+\partial_{t^{\prime}} v\left(t^{\prime}\right)\right] \\
& \left.=S_{\beta}\left(t-t^{\prime}\right)\left(-\left(\partial_{x} v\right)^{2}\right)\left(t^{\prime}\right)\right)
\end{aligned}
$$

integrando y aplicando el segundo teorema fundamental del cálculo, se obtiene

$$
\begin{aligned}
\int_{0}^{t} \partial_{t^{\prime}}\left(S_{\beta}\left(t-t^{\prime}\right) v\left(t^{\prime}\right)\right) d t^{\prime} & =-\int_{0}^{t} S_{\beta}\left(t-t^{\prime}\right)\left(\partial_{x} v\right)^{2}\left(t^{\prime}\right) d t^{\prime} \\
v(t) & =S_{\beta}(t) v(0)-\int_{0}^{t} S_{\beta}\left(t-t^{\prime}\right)\left(\partial_{x} v\right)^{2}\left(t^{\prime}\right) d t^{\prime} \\
v(t) & =S_{\beta}(t) \varphi-\int_{0}^{t} S_{\beta}\left(t-t^{\prime}\right)\left(\partial_{x} v\right)^{2}\left(t^{\prime}\right) d t^{\prime},
\end{aligned}
$$

para $0<t^{\prime}<t$.

2. Recíprocamente, si asumimos que $v \in C\left([0, T] ; H_{\text {per }}^{s}\right)$ es solución de la ecuación integral 11. Sabemos que $\partial_{t} v(t)=\partial_{t} S_{\beta}(t) \phi-\partial_{t} \int_{0}^{t} S_{\beta}\left(t-t^{\prime}\right)\left(\partial_{x} v\right)^{2}\left(t^{\prime}\right) d t^{\prime}=Q_{\beta}(D) S_{\beta}(t) \phi-$ $\partial_{t} \int_{0}^{t} S_{\beta}\left(t-t^{\prime}\right)\left(\partial_{x} v\right)^{2}\left(t^{\prime}\right) d t^{\prime}$. Por lo tanto, para demostrar que 11) satisface 10 , queda por calcular $\partial_{t} \int_{0}^{t} S_{\beta}\left(t-t^{\prime}\right)\left(\partial_{x} v\right)^{2}\left(t^{\prime}\right) d t^{\prime}$. 
Sea $t>0$ fijo y tomando $h>0$. Entonces

$$
\begin{aligned}
\lim _{h \rightarrow 0} \frac{1}{h}\left[\int_{0}^{t+h} S_{\beta}(t\right. & \left.\left.+h-t^{\prime}\right)\left(\partial_{x} v\right)^{2}\left(t^{\prime}\right) d t^{\prime}-\int_{0}^{t} S_{\beta}\left(t-t^{\prime}\right)\left(\partial_{x} v\right)^{2}\left(t^{\prime}\right) d t^{\prime}\right] \\
= & \lim _{h \rightarrow 0} \frac{1}{h} \int_{0}^{t}\left(S_{\beta}\left(t+h-t^{\prime}\right)-S_{\beta}\left(t-t^{\prime}\right)\right)\left(\partial_{x} v\right)^{2}\left(t^{\prime}\right) d t^{\prime} \\
& +\lim _{h \rightarrow 0} \frac{1}{h} \int_{t}^{t+h} S_{\beta}\left(t-t^{\prime}\right)\left(\partial_{x} v\right)^{2}\left(t^{\prime}\right) d t^{\prime} .
\end{aligned}
$$

La segunda integral en el lado derecho de $(12)$ es el valor medio de una función continua (teorema 7) en un intervalo que se reduce a cero cuando $h \rightarrow 0$. Debe converger al integrando en $t$, esto es,

$$
\lim _{h \rightarrow 0}\left\|\frac{1}{h} \int_{t}^{t+h} S_{\beta}\left(t+h-t^{\prime}\right)\left(\partial_{x} v\right)^{2}\left(t^{\prime}\right) d t^{\prime}-\left(\partial_{x} v\right)^{2}(t)\right\|_{s-4}=0 .
$$

Ahora por la convergencia uniforme establecida en el teorema 5, se sigue que

$$
\begin{aligned}
& \lim _{h \rightarrow 0} \frac{1}{h} \int_{0}^{t}\left[S_{\beta}\left(t+h-t^{\prime}\right)-S_{\beta}\left(t-t^{\prime}\right)\right]\left(\partial_{x} v\right)^{2}\left(t^{\prime}\right) d t^{\prime} \\
& =\int_{0}^{t} \partial_{t} S_{\beta}\left(t-t^{\prime}\right)\left(\partial_{x} v\right)^{2}\left(t^{\prime}\right) d t^{\prime} \\
& =Q_{\beta}(D) \int_{0}^{t} S_{\beta}\left(t-t^{\prime}\right)\left(\partial_{x} v\right)^{2}\left(t^{\prime}\right) d t^{\prime}
\end{aligned}
$$

donde el límite es tomado en $H_{\text {per }}^{s-4}$. Usando 11 podemos reescribir 14 como

$$
\lim _{h \rightarrow 0} \frac{1}{h} \int_{0}^{t}\left[S_{\beta}\left(t+h-t^{\prime}\right)-S_{\beta}\left(t-t^{\prime}\right)\right]\left(\partial_{x} v\right)^{2}\left(t^{\prime}\right) d t^{\prime}=Q_{\beta}(D)\left\{-v(t)+S_{\beta}(t) \varphi\right\} .
$$

En consecuencia, de (12), usando (13) y $\left(14^{\prime}\right)$ obtenemos

$$
\begin{aligned}
\partial_{t} v(t) & =\partial_{t} S_{\beta}(t) \varphi-\partial_{t} \int_{0}^{t} S_{\beta}\left(t-t^{\prime}\right)\left(\partial_{x} v\right)^{2}\left(t^{\prime}\right) d t^{\prime} \\
& =Q_{\beta}(D) S_{\beta}(t) \varphi-\left(\partial_{x} v\right)^{2}(t)+Q_{\beta}(D)\left\{v(t)-S_{\beta}(t) \varphi\right\} \\
& =Q_{\beta}(D) v(t)-\left(\partial_{x} v\right)^{2}(t) .
\end{aligned}
$$

Nuestro siguiente paso es probar la existencia de una solución. Definimos para $T>0, s>$ $3 / 2, M>0$ у $\varphi \in H_{p e r}^{s}$ el espacio:

$$
\Lambda_{s}(T, M, \varphi)=\left\{v \in C\left([0, T] ; H_{p e r}^{s}\right) /\left\|v(t)-S_{\beta}(t) \varphi\right\|_{s} \leq M, \forall t \in[0, T]\right\}
$$

dotado de la métrica

$$
d(v, w)=\underset{[0, T]}{\sup \|v(t)-w(t)\|_{s}}
$$

es un espacio métrico completo. Consideremos la aplicación

$$
A(v)(t)=\underbrace{S_{\beta}(t) \varphi}_{\in H_{\text {per }}^{s}}-\underbrace{\int_{0}^{t} S_{\beta}\left(t-t^{\prime}\right)\left(\partial_{x} v\right)^{2}\left(t^{\prime}\right) d t^{\prime}}_{I_{1}:=}
$$


Proposición 3. Sea $s>3 / 2, v \in C\left([0, T] ; H_{\text {per }}^{s}\right)$, entonces, $A(v) \in C\left([0, T] ; H_{\text {per }}^{s}\right)$.

Demostración. 1. Primeramente probaremos que para $t>0$ fijado, se tiene que $A(v)(t) \in$ $H_{\text {per }}^{s}, \forall t \geq 0$.

Usando la estimativa de regularización, teorema 6 , para $\lambda=1$ se tiene

$$
\begin{aligned}
\left\|I_{1}\right\|_{s} & \leq \int_{0}^{t} K_{1}\left(1+\frac{1}{\sqrt[4]{\beta\left(t-t^{\prime}\right)}}\right)\left\|\left(\partial_{x} v\right)^{2}\left(t^{\prime}\right)\right\|_{s-1} d t^{\prime}, s>3 / 2 \\
& \leq \int_{0}^{t} K_{1}\left(1+\frac{1}{\sqrt[4]{\beta\left(t-t^{\prime}\right)}}\right)\left\|v\left(t^{\prime}\right)\right\|_{s}^{2} d t^{\prime} \\
& \leq K_{1} \sup _{t^{\prime} \in[0, T]}\left\|v\left(t^{\prime}\right)\right\|_{s}^{2} \int_{0}^{t}\left(1+\frac{1}{\sqrt[4]{\beta\left(t-t^{\prime}\right)}}\right) d t^{\prime} \\
& \leq K^{\prime} \sup _{t^{\prime} \in[0, T]}\left\|v\left(t^{\prime}\right)\right\|_{s}^{2} \int_{0}^{t} \frac{1}{\sqrt[4]{\left(t-t^{\prime}\right)}} d t^{\prime} \\
& \leq K_{t^{\prime} \in[0, T]} \sup ^{\prime}\left\|v\left(t^{\prime}\right)\right\|_{s}^{2} T^{3 / 4}<\infty .
\end{aligned}
$$

Luego $I_{1} \in H_{\text {per }}^{s}$. Ahora como $S_{\beta}(t) \phi \in H_{\text {per }}^{s}$, entonces $A v(t) \in H_{\text {per }}^{s}$, para $t>0$. El caso $t=0, A v(0) \in H_{p e r}^{s}$. Por lo tanto $A v(t) \in H_{p e r}^{s}, \forall t \geq 0$.

2. Ahora veamos la continuidad de $A v(\cdot)$. Como ya está establecida la continuidad del semigrupo $S_{\beta}(t) \varphi$ en $H_{p e r}^{s}\left(C_{0}\right.$-semigrupo), basta ver la continuidad de $G, G(t):=\int_{0}^{t} S_{\beta}(t-$ $\left.t^{\prime}\right)\left(\partial_{x} v\right)^{2}\left(t^{\prime}\right) d t^{\prime}$. Tomando $h>0$, se tiene

$$
\begin{aligned}
G(t+h)-G(t) & =\int_{0}^{t+h} S_{\beta}\left(t+h-t^{\prime}\right)\left(\partial_{x} v\right)^{2}\left(t^{\prime}\right) d t^{\prime}-\int_{0}^{t} S_{\beta}\left(t-t^{\prime}\right)\left(\partial_{x} v\right)^{2}\left(t^{\prime}\right) d t^{\prime} \\
& =\int_{0}^{t}\left[S_{\beta}\left(t+h-t^{\prime}\right)-S_{\beta}\left(t-t^{\prime}\right)\right]\left(\partial_{x} v\right)^{2}\left(t^{\prime}\right) d t^{\prime} \\
& \left.+\int_{t}^{t+h} S_{\beta}\left(t+h-t^{\prime}\right)\right]\left(\partial_{x} v\right)^{2}\left(t^{\prime}\right) d t^{\prime} \\
& =\underbrace{\left(S_{\beta}(h)-I\right) \int_{0}^{t} S_{\beta}\left(t-t^{\prime}\right)\left(\partial_{x} v\right)^{2}\left(t^{\prime}\right) d t^{\prime}}_{I_{3}(h):=}+\underbrace{\int_{t}^{t+h} S_{\beta}\left(t+h-t^{\prime}\right)\left(\partial_{x} v\right)^{2}\left(t^{\prime}\right) d t^{\prime}}_{I_{2}(h):=}
\end{aligned}
$$

la segunda integral en (18) cumple la siguiente desigualdad

$$
\left\|I_{2}(h)\right\|_{s}=\left\|\int_{t}^{t+h} S_{\beta}\left(t+h-t^{\prime}\right)\left(\partial_{x} v\right)^{2}\left(t^{\prime}\right) d t^{\prime}\right\|_{s} \leq \int_{t}^{t+h}\left\|S_{\beta}\left(t+h-t^{\prime}\right)\left(\partial_{x} v\right)^{2}\left(t^{\prime}\right)\right\|_{s} d t^{\prime} .
$$

Aplicando la estimativa de regularización Teorema 6 , para $\lambda=1$, se tiene en $(19)$

$$
\begin{aligned}
\int_{t}^{t+h}\left\|S_{\beta}\left(t+h-t^{\prime}\right)\left(\partial_{x} v\right)^{2}\left(t^{\prime}\right)\right\|_{s} d t^{\prime} & \leq K_{1} \int_{t}^{t+h}\left(1+\frac{1}{\sqrt[4]{\beta\left(t+h-t^{\prime}\right)}}\right)\left\|\left(\partial_{x} v\right)^{2}\left(t^{\prime}\right)\right\|_{s-1} d t^{\prime} \\
& \leq K \sup _{t^{\prime} \in[0, T]}\left\|v\left(t^{\prime}\right)\right\|_{s}^{2} \int_{t}^{t+h} \frac{1}{\sqrt[4]{t+h-t^{\prime}}} d t^{\prime} \\
& \leq K \sup _{t^{\prime} \in[0, T]}\left\|v\left(t^{\prime}\right)\right\|_{s}^{2} h^{3 / 4} .
\end{aligned}
$$


Así, $\|G(t+h)-G(t)\|_{s} \leq\left\|I_{3}(h)\right\|_{s}+\left\|I_{2}(h)\right\|_{s} \rightarrow 0$ cuando $h \rightarrow 0^{+}$.

Análogamente se obtiene la continuidad a izquierda.

Proposición 4. Sea $s>3 / 2$, entonces existe un $T>0$ tal que $A: \Lambda_{s}(T, M, \varphi) \rightarrow \Lambda_{s}(T, M, \varphi)$ es una contracción.

Demostración. 1. Veamos que existe $T_{1}>0$ tal que $A: \Lambda_{s}\left(T_{1}, M, \varphi\right) \rightarrow \Lambda_{s}\left(T_{1}, M, \varphi\right)$. Sea $v \in \Lambda_{s}(T, M, \varphi)$, entonces

$$
\begin{aligned}
\left\|A(v)(t)-S_{\beta}(t) \varphi\right\|_{s} & =\left\|-\int_{0}^{t} S_{\beta}\left(t-t^{\prime}\right)\left(\partial_{x} v\right)^{2}\left(t^{\prime}\right) d t^{\prime}\right\|_{s} \\
& \leq \int_{0}^{t}\left\|S_{\beta}\left(t-t^{\prime}\right)\left(\partial_{x} v\right)^{2}\left(t^{\prime}\right)\right\|_{s} d t^{\prime} .
\end{aligned}
$$

Acotamos (20) usando la estimativa de regularización (teorema 6) con $\lambda=1$ y aplicando el lema de inmersión de Sobolev, ya que $s>\frac{3}{2}$. En efecto,

$$
\begin{aligned}
\left\|S_{\beta}\left(t-t^{\prime}\right)\left(\partial_{x} v\right)^{2}\left(t^{\prime}\right)\right\|_{s} & =\left\|S_{\beta}\left(t-t^{\prime}\right)\left(\partial_{x} v\right)^{2}\left(t^{\prime}\right)\right\|_{s-1+1} \\
& \leq K_{1}\left[1+\frac{1}{\sqrt[4]{\beta\left(t-t^{\prime}\right)}}\right]\left\|\left(\partial_{x} v\right)^{2}\left(t^{\prime}\right)\right\|_{s-1} \\
& \leq K_{1}\left[1+\frac{1}{\sqrt[4]{\beta\left(t-t^{\prime}\right)}}\right]\left\|v\left(t^{\prime}\right)\right\|_{s}^{2}
\end{aligned}
$$

La desigualdad (21) y $\left\|v\left(t^{\prime}\right)-S_{\beta}\left(t^{\prime}\right) \varphi\right\|_{s} \leq M$ convierten a (20) en

$$
\begin{aligned}
\left\|A(v)(t)-S_{\beta}(t) \varphi\right\|_{s} & \leq K_{1}\left(M+\|\varphi\|_{s}\right)^{2} \int_{o}^{t}\left[1+\frac{1}{\sqrt[4]{\beta\left(t-t^{\prime}\right)}}\right] d t^{\prime} \\
& \leq K\left(M+\|\varphi\|_{s}\right)^{2} T^{3 / 4} .
\end{aligned}
$$

Ahora, tomamos $T_{1}>0$ en 22 tal que

$$
\left.K\left(M+\|\varphi\|_{s}\right)^{2} T_{1}^{3 / 4}\right) \leq M .
$$

Así, hemos probado que $A\left(\Lambda_{s}(T, M, \varphi)\right) \subseteq \Lambda_{s}(T, M, \varphi)$ para cada $T \leq T_{1}$.

2. Veamos ahora que existe $T_{2}$ tal que $A$ es una contracción en $\Lambda_{s}\left(T_{2}, M, \varphi\right)$. Sean $v, w \in$ $\Lambda_{s}(T, M, \varphi)$, debemos probar que

$$
\exists \alpha \in(0,1), d(A v, A w) \leq \alpha d(v, w) . \forall v, w \in \Lambda_{s}\left(T_{2}, M, \varphi\right) .
$$

Considerando nuevamente la estimativa de regularización con $\lambda=1$. Sea $s>3 / 2$ y $v, w \in$ $\Lambda_{s}(T, M, \varphi)$,

$$
\begin{aligned}
\| A(v)(t) & -A(w)(t) \|_{s} \\
& \leq \int_{0}^{t}\left\|S_{\beta}\left(t-t^{\prime}\right)\left(\left(\partial_{x} v\right)^{2}\left(t^{\prime}\right)-\left(\partial_{x} w\right)^{2}\left(t^{\prime}\right)\right)\right\|_{s} d t^{\prime} \\
& \leq \int_{0}^{t} K_{1}\left[1+\left(\frac{1}{\beta\left(t-t^{\prime}\right)}\right)^{\frac{1}{4}}\right]\left\|\left(\partial_{x} v\right)^{2}\left(t^{\prime}\right)\left(t^{\prime}\right)-\left(\partial_{x} w\right)^{2}\left(t^{\prime}\right)\right\|_{s-1} d t^{\prime},
\end{aligned}
$$


donde $A(v), A(w) \in \Lambda_{s}(T, M, \varphi)$. La desigualdad

$$
\begin{aligned}
\left\|\left(\partial_{x} v\right)^{2}\left(t^{\prime}\right)-\left(\partial_{x} w\right)^{2}\left(t^{\prime}\right)\right\|_{s-1} & \leq\left\|\partial_{x} v\left(t^{\prime}\right)-\left(\partial_{x} w\right)\left(t^{\prime}\right)\right\|_{s-1}\left\|\partial_{x} v\left(t^{\prime}\right)+\left(\partial_{x} w\right)\right\|_{s-1} \\
& \leq\left\|v\left(t^{\prime}\right)-w\left(t^{\prime}\right)\right\|_{s}\left\|v\left(t^{\prime}\right)+w\left(t^{\prime}\right)\right\|_{s} \\
& \leq K_{s}\left\|v\left(t^{\prime}\right)-w\left(t^{\prime}\right)\right\|_{s}\left(\left\|v\left(t^{\prime}\right)\right\|_{s}+\left\|w\left(t^{\prime}\right)\right\|_{s}\right) \\
& \leq 2 K_{s}\left(M+\|\varphi\|_{s}\right) d(v, w)
\end{aligned}
$$

es consecuencia de $\left\|v\left(t^{\prime}\right)\right\|_{s}=\left\|v\left(t^{\prime}\right)-S_{\beta}(t) \varphi+S_{\beta}(t) \varphi\right\|_{s} \leq\left(M+\|\varphi\|_{s}\right), \partial_{x} \in \mathcal{B}\left(H_{\text {per }}^{s}, H_{\text {per }}^{s-1}\right)$ y que $H^{s}$ es un álgebra de Banach para $s>3 / 2$.

De (24) :

$$
\begin{aligned}
\|A v(t)-A w(t)\|_{s} & \leq K_{1} K_{s}\left(M+\|\varphi\|_{s}\right) d(v, w) \int_{0}^{t}\left[1+\left(\frac{1}{\beta\left(t-t^{\prime}\right)}\right)^{\frac{1}{4}}\right] d t^{\prime} \\
& \leq K\left(M+\|\varphi\|_{s}\right) d(v, w) T^{3 / 4}
\end{aligned}
$$

Luego, elegimos $T_{2}>0$, tal que

$$
K\left(M+\|\varphi\|_{s}\right) T_{2}^{3 / 4}<1 .
$$

Así, $A$ es una contracción. Por lo tanto, si $\widetilde{T}=\min \left\{T_{1}, T_{2}\right\}$ se tiene que la aplicación $A: \Lambda_{s}(T, M, \varphi) \rightarrow \Lambda_{s}(T, M, \varphi)$ es una contracción cuando $0<T \leq \widetilde{T}$.

Observación 3. Hemos obtenido, gracias al teorema del punto fijo de Banach, que existen $T>0$ y $v \in C\left([0, T] ; H_{p e r}^{s}\right)$ tal que

$$
v(t)=A(v)(t)=S_{\beta}(t) \varphi-\int_{0}^{t} S_{\beta}\left(t-t^{\prime}\right)\left(\partial_{x} v\right)^{2}\left(t^{\prime}\right) d t^{\prime} .
$$

Por lo tanto, la proposición (3) y la proposición (4) implican que (1) tiene solución en $H_{\text {per }}^{s}$, para $s>3 / 2$.

A continuación veamos la unicidad.

Teorema 9. Sea $\varphi \in H_{\text {per }}^{s}$, con $s>3 / 2$. Entonces existe una única solución $v \in C\left([0, T] ; H_{p e r}^{s}\right)$ de la ecuación integral (11).

Demostración. Sean $v$ y $w$ soluciones de $(1)$ tal que $v(0)=\varphi$ y $w(0)=\psi \in H_{\text {per }}^{s}$, gracias a la ecuación integral (11), la desigualdad triangular y la estimativa de regularización Teorema 6 , para $\lambda=1$ y $s>3 / 2$ se obtiene

$$
\begin{aligned}
\| v(t) & -w(t) \|_{s} \\
& \leq\left\|S_{\beta}(t)(\varphi-\psi)\right\|_{s}+\int_{o}^{t}\left\|S_{\beta}\left(t-t^{\prime}\right)\left(\left(\partial_{x} v\right)^{2}\left(t^{\prime}\right)-\left(\partial_{x} w\right)^{2}\left(t^{\prime}\right)\right)\right\|_{s} \\
& \leq\|\varphi-\psi\|_{s}+\int_{o}^{t} K_{1}\left[1+\left(\frac{1}{\beta\left(t-t^{\prime}\right)}\right)^{\frac{1}{4}}\right]\left\|\left(\partial_{x} v\right)^{2}\left(t^{\prime}\right)-\left(\partial_{x} w\right)^{2}\left(t^{\prime}\right)\right\|_{s-1} d t .
\end{aligned}
$$

Eligiendo $T_{s}$ si es necesario, para que $\frac{1}{\sqrt[4]{\beta\left(t-t^{\prime}\right)}} \geq 1$, (25) se transforma en

$$
\begin{aligned}
\|v(t)-w(t)\|_{s} & \\
& \leq\|\varphi-\psi\|_{s}+K\left(1+\|\varphi\|_{s}+\|\psi\|_{s}\right) \int_{0}^{t} \frac{1}{\sqrt[4]{\left(t-t^{\prime}\right)}}\left\|v\left(t^{\prime}\right)-w\left(t^{\prime}\right)\right\|_{s} d t^{\prime} .
\end{aligned}
$$

De (26), usando el lema 1 con $g(t)=\|v(t)-w(t)\|_{s}, a=\|\varphi-\psi\|_{s}, b=K\left(1+\|\varphi\|_{s}+\|\psi\|_{s}\right), \beta=\frac{3}{4}$ y $\gamma=1$ obtenemos que si $\psi=\varphi \Rightarrow v=w$. 
Seguidamente enunciamos el resultado de la continuidad de la solución $v$ respecto al dato inicial $\varphi$. Con este ultimo resultado obtendríamos el objetivo final del trabajo, es decir, el buen planteamiento local de la KdV-K-S.

Teorema 10. Sea $\varphi_{n} \in H_{p e r}^{s}, n=1,2, \ldots, \varphi$ tal que $\varphi_{n} \stackrel{H_{p e r}^{s}}{\longrightarrow} \varphi_{\infty}$. Sea $v_{n} \in C\left(\left[0, \widetilde{T}_{n}\right) ; H_{p e r}^{s}\right)$, donde $\widetilde{T}_{n}=\widetilde{T}\left(M,\left\|\varphi_{n}\right\|_{s}\right)$, soluciones obtenidas en el teorema 9. Sea $T \in(0, \widetilde{T} \infty)$. Entonces las soluciones $v_{n}$ se definen en $[0 ; T]$ para $n$ suficientemente grande $y$

$$
\lim _{n \rightarrow \infty} \sup _{t \in[0, T]}\left\|v_{n}(t)-v_{\infty}(t)\right\|_{s}=0
$$

Demostración. La demostración de la proposición (4) implica que el tiempo de existencia $\widetilde{T}\left(M,\|\varphi\|_{s}\right)$ es una función continua de $\varphi$, luego existe $N \in \mathbb{N}$ tal que $\widetilde{T}_{n}>T$ para todo $n \geq N$. Así $v_{n}$ se define en $[0, T]$ para tales $n$. Como $v_{n}, n=1,2, \ldots$ es solución de (1) con dato inicial $v_{n}(0)=\varphi_{n}, n=1,2, \ldots$, se sigue que $v_{n} \in \Lambda_{s}\left(T, M, \varphi_{n}\right)$ para todo $n \geq N$ y satisface

$$
\left\|v_{n}(t)\right\|_{s} \leq\left\|\varphi_{n}\right\|_{s}+M \leq R+M
$$

donde $R=\sup _{n}\left\|\varphi_{n}\right\|$. Como $s>3 / 2$, combinamos (26) con (27) para obtener

$$
\left\|v_{n}(t)-v_{\infty}(t)\right\|_{s} \leq\left\|\varphi_{n}-\varphi_{\infty}\right\|_{s}+K(R+M) \int_{0}^{t} \frac{1}{\sqrt[4]{\left(t-t^{\prime}\right)}}\left\|v_{n}\left(t^{\prime}\right)-v_{\infty}\left(t^{\prime}\right)\right\|_{s} d t^{\prime}
$$

y el resultado se sigue de (28) y el lema 1.

Por último obtendremos mas regularidad de la solución usando estimativa de regularización Teorema 6 en los espacios de Sobolev periódico.

Proposición 5. Sea $v \in C\left([0, T] ; H_{p e r}^{s}\right), s>\frac{3}{2}$ que satisface (1) con dato inicial $v(0)=\varphi \in H_{\text {per }}^{s}$ obtenida en el teorema (9), entonces $v \in C\left([0, T] ; H_{\text {per }}^{r}\right)$ para $r>s$. Además $v \in C([0, T] ; \mathcal{P})$.

Demostración. Si $0<\lambda<3$ y $s>3 / 2$ por la estimativa de regularización Teorema 6 con $s-1>0$ en lugar de $s$ y $\lambda+1>0$ en lugar de $\lambda$, implican que,

$$
\begin{aligned}
\|v(t)\|_{s+\lambda} & \leq\left\|S_{\beta}(t) \varphi\right\|_{s+\lambda}+\int_{0}^{t}\left\|S_{\beta}\left(t-t^{\prime}\right)\left(\partial_{x} v\right)^{2}\left(t^{\prime}\right)\right\|_{s+\lambda} d t^{\prime} \\
& \leq K_{\lambda}\left(1+\frac{1}{(\beta t)^{\frac{\lambda+1}{4}}}\right)\|\varphi\|_{s-1}+K_{\lambda} \int_{0}^{t}\left(1+\frac{1}{\left(\beta\left(t-t^{\prime}\right)\right)^{\frac{\lambda+1}{4}}}\right)\left\|\left(\partial_{x} v\right)^{2}\left(t^{\prime}\right)\right\|_{s-1} d t^{\prime} \\
& \leq K_{\lambda}\left(1+\frac{1}{(\beta t)^{\frac{\lambda+1}{4}}}\right)\|\varphi\|_{s}+K_{\lambda} \int_{0}^{t}\left(1+\frac{1}{\left(\beta\left(t-t^{\prime}\right)\right)^{\frac{\lambda+1}{4}}}\right)\left\|v\left(t^{\prime}\right)\right\|_{s}^{2} d t^{\prime} \\
& \leq K_{\lambda}\left(1+\frac{1}{(\beta t)^{\frac{\lambda+1}{4}}}\right)\|\varphi\|_{s}+K_{\lambda} \sup _{t^{\prime} \in[0, T]}\left\|v\left(t^{\prime}\right)\right\|_{s}^{2} \int_{0}^{t}\left(1+\frac{1}{\left(\beta\left(t-t^{\prime}\right)\right)^{\frac{1+\lambda}{4}}}\right) d t^{\prime}
\end{aligned}
$$

como la integral de 29 es finita, tenemos que $v \in C\left([0, T] ; H_{p e r}^{s+\lambda}\right)$. Con este argumento obtenemos lo requerido.

\section{Conclusión}

1. Usando la estimativa de regularización del semigrupo asociado a la parte lineal, y aplicando el teorema del punto fijo de Banach, fue posible obtener la existencia de solución de la KdV-K-S no lineal. 
2. Fué posible obtener la regularidad de la solución de la KdV-K-S no lineal usando estimativas del semigrupo asociado a la parte lineal.

3. Gracias a la transformada de Fourier obtuvimos formal y explicitamente la solución de la parte lineal y es gracias a ello que podemos introducir el semigrupo asociado que fué de gran ayuda en todo nuestro estudio.

4. Finalmente, este trabajo de investigación contribuye y motiva al desarrollo de este campo creciente de aplicaciones de la física-matemática, específicamente en la dinámica de fluidos viscosos.

\section{Referencias bibliográficas}

[1] Biagioni, H.A., Bona, J.L., Iorio, R. and Scialom.(1996). On the Korteweg de Vries Kuramoto Sivashinsky equation. Adv. Diff. Eq., 1(1): 1-20.

[2] Henry, D.(1981). Geometric Theory of Semilinear Parabolic Equation. Lecture Notes in Mathematics 840, Springer Verlag, Berlin.

[3] Rafael José Iorio, Jr and Valéria de Magalhães Iorio.(2002). Fourier Analysis and partial Differential Equations. Cambridge University Press.

[4] Kreyszig, E.(1978). Introductory Functional Analysis With Applications. University of Windsor. Jhon Wiley \& Sons. Inc.

[5] Liu, Z. and Zheng, S.(1999). Semigroups associated with dissipative system. Chapman \& Hall/CRC.

[6] Pazy, A.(1983). Semigruops of linear Operator and applications to partial differential equations. Appl. Math. Sci, 44 Springer Verlag. Berlin.

[7] Santiago Ayala, Yolanda and Rojas, Santiago.(2017). Regularity and wellposedness of a problem to one parameter and its behavior at the limit. Bulletin of the Allahabad Mathematical Society 32 (02): 207-230.

[8] Tadmor E.(1986). The wellposedness of the Kuramoto Sivashisnky equation. SIAM J. Math. Anal. 17 page $884-893$.

[9] Topper, J. and Kawahara, T.(1978). Approximate equations for long nonlinear waves on a viscous fluid. J. Phys. Soc. Japan, 44: 663-666. 